\title{
FAMILIES OF CURVES CONFORMALLY EQUIVALENT TO CIRCLES
}

\author{
BY \\ EDWARD KASNER AND JOHN DE CICCO
}

1. Introduction. In this paper, we shall study three-parameter families of curves conformally equivalent to the totality of circles. We obtain the analytic form and several complete geometric characterizations of any such family, which we shall call an $\Omega$ family.

In minimal coordinates $(u, v)$ the differential equation of such a family is of the form

$$
2 \frac{d v}{d u} \frac{d^{3} v}{d u^{3}}-3\left(\frac{d^{2} v}{d u^{2}}\right)^{2}=2\left(\frac{d v}{d u}\right)^{2}\left[A(u)-\left(\frac{d v}{d u}\right)^{2} B(v)\right] .
$$

Our fundamental result is that any $\Omega$ family may be characterized among all three-parameter families of curves by the following three properties: Property I. The locus of the foci of the osculating parabolas of the $\infty^{1}$ curves which contain a given lineal element $E$ is a lemniscate $L$ with $E$ as one of the two orthogonal tangent elements at the node of $L$. Property II. As the direction of $E$ is rotated about its point $P$, the locus of the centers of the orthogonal pairs of circles defining the $\infty^{1}$ focal lemniscates is an equilateral hyperbola $H$ with the center of $H$ at $P$. Property III. The foci of the equilateral hyperbolas are connected to the point $P$ by a direct conformal transformation.

Another complete geometric characterization of an $\Omega$ family of curves is the following. Property $\mathrm{I}^{\prime}$. The envelope of the directrices of the osculating parabolas of the $\infty^{1}$ curves which contain a given lineal element $E$ is an equilateral hyperbola $H$ with the point of $E$ as the center of $H$ and the line of $E$ as one of the asymptotes of $H$. Property II'. The locus of the foci of the directorial equilateral hyperbolas is an equilateral hyperbola $H^{\prime}$ with its center at $P$. Property III'. The four foci of the equilateral hyperbola $H^{\prime}$ of Property II' are related to the point $P$ by a direct conformal transformation.

A reciprocity relation appears in these two sets of geometric characterizations. But this reciprocal relation is by no means self-evident. For although the two characterizations are roughly dual, separate proofs are required. The lemniscate and the equilateral hyperbola of Properties I and I' are equivalent under inversion, whereas the equilateral hyperbolas of Properties II and II' are equivalent under a similitude.

In connection with this duality, the following results may be noted. The $\infty^{1}$ focal lemniscates of Property I, constructed at the point $P$, all pass

Presented to the Society, April 27, 1940; received by the editors April 10, 1940. 
through the vertices of a minimal quadrilateral with center at $P$. The $\infty^{1}$ directorial equilateral hyperbolas of Property $\mathrm{I}^{\prime}$, constructed at $P$, are tangent to the sides of a quadrilateral whose diagonals are the minimal lines through $P$.

In the latter part of our paper, we shall give other geometric properties of an $\Omega$ family of curves. The rate $\kappa^{\prime}$ of variation of the curvature with respect to the arc length is the same for all the curves of our family which contain a lineal element $E$. The resulting $\infty^{1}$ rates of variation of curvature at a point $P$ are connected by an analogue of Meusnier's theorem. We note that our problem may be considered to be a generalization of the family of curves conformally equivalent to the $\infty^{2}$ straight lines. This simple type has been considered elsewhere. Relations to dynamical and natural families are of interest.

2. The differential equation of any $\Omega$ family. For the analytic work, we shall find it convenient to use the minimal coordinates $(u, v)$ instead of the ordinary rectangular cartesian coordinates $(x, y)$. These are connected by the relations

$$
u=x+i y, \quad v=x-i y .
$$

An $\Omega$ family of curves consists of a three-parameter set which is equivalent to the $\infty^{3}$ circles under a given conformal transformation. We proceed to derive the differential equation of any such family. However, before this can be accomplished, it is necessary to discuss some preliminary material.

In minimal coordinates $(u, v)$, any direct conformal transformation is

$$
U=\phi(u), \quad V=\psi(v),
$$

where the functions are of course analytic. A reverse conformal transformation may be expressed as the product of a direct conformal transformation by the reflection through the $x$-axis: $U=v, V=u$. Upon extending this conformal transformation three times, we find

$$
\begin{aligned}
P= & \frac{\psi_{v}}{\phi_{u}} p, \\
P^{\prime}= & \frac{\psi_{v}}{\phi_{u}^{2}} p^{\prime}+\frac{\psi_{v v}}{\phi_{u}^{2}} p^{2}-\frac{\psi_{v} \phi_{u u}}{\phi_{u}^{3}} p, \\
P^{\prime \prime}= & \frac{\psi_{v}}{\phi_{u}^{3}} p^{\prime \prime}+\frac{3 \psi_{v v}}{\phi_{u}^{3}} p p^{\prime}-\frac{3 \psi_{v} \phi_{u u}}{\phi_{u}^{4}} p^{\prime}+\frac{\psi_{v v v}}{\phi_{u}^{3}} p^{3}-\frac{3 \psi_{v v} \phi_{u u}}{\phi_{u}^{4}} p^{2} \\
& +\frac{\psi_{v}}{\phi_{u}^{5}}\left(-\phi_{u} \phi_{u u u}+3 \phi_{u u}^{2}\right) p,
\end{aligned}
$$

where $p=d v / d u$ and $P=d V / d U$.

The $\infty^{3}$ circles of the plane are represented in minimal coordinates by the $\infty^{3}$ conics which pass through the two fixed points at infinity, given in homo- 
geneous minimal coordinates by $(0,1,0)$ and $(1,0,0)$. Therefore the equation of any circle is

$$
a u v+b u+c v+d=0 .
$$

From this, the differential equation of all $\infty^{3}$ circles is found to be

$$
2 p p^{\prime \prime}-3 p^{\prime 2}=0 \text {. }
$$

The Moebius group of the complex plane is the group of all point transformations which preserve the entire family of $\infty^{3}$ circles. In minimal coordinates, any direct Moebius transformation may be written as

$$
U=\frac{a u+b}{c u+d}, \quad V=\frac{a^{\prime} v+b^{\prime}}{c^{\prime} v+d^{\prime}} .
$$

A reverse Moebius transformation is the product of a direct Moebius transformation by the reflection through the $x$-axis. A Moebius transformation is said to be real if the coefficients of one of the above equations are the respective conjugates of those of the other. In the complex cartesian plane, there are $2 \infty^{6}$ Moebius transformations, of which $2 \infty^{3}$ are real.

Upon applying the conformal transformation (2) to the circles of the $(u, v)$ plane, the differential equation (5) expressed in capital letters is carried into the differential equation

$$
2 p p^{\prime \prime}-3 p^{\prime 2}=2 p^{2}\left(A-B p^{2}\right)
$$

where

$$
A=\frac{2 \phi_{u} \phi_{u u u}-3 \phi_{u u}^{2}}{2 \phi_{u}^{2}}, \quad B=\frac{2 \psi_{v} \psi_{v v v}-3 \psi_{v v}^{2}}{2 \psi_{v}^{2}} .
$$

From this, it is found that $A$ may be any function of $u$ only and $B$ may be any function of $v$ only. (All our functions in this paper are assumed to be analytic.)

THEOREM 1. A differential equation of the third order represents an $\Omega$ family of curves if and only if it is of the form (where $p=v^{\prime}=d v / d u$ )

$$
2 p p^{\prime \prime}-3 p^{\prime 2}=2 p^{2}\left(A-B p^{2}\right),
$$

where $A$ is an arbitrary function of $u$ and $B$ is an arbitrary function of $v$.

By the preceding differential equation, we observe that any $\Omega$ family is uniquely determined by the functions $A$ and $B$. Therefore $\Omega$ is a function of $A$ and $B$ only and we write $\Omega=\Omega(A, B)$.

If $\phi(u)$ and $\psi(v)$ are any two functions which satisfy (8), then the integral curves of (9) are the transforms under the conformal transformation (2) of the $\infty^{3}$ circles of the $(U, V)$ plane. Therefore the curves of our $\Omega$ family are

$$
a \phi \psi+b \phi+c \psi+d=0 \text {. }
$$


Any $\Omega$ family of curves is thus a special type of linear families of curves. Of course, the differential equation (9) could have been obtained as a result of eliminating the arbitrary constants from the above equation by differentiation.

By (10), it is obvious that under a conformal transformation any $\Omega$ family is converted in general into some other $\Omega$ family. The group of transformations which preserve a given $\Omega(A, B)$ family of curves is a mixed six-parameter group $G_{6}^{\prime}[\Omega(A, B)]$ isomorphic with the Moebius group of circular transformations. Any transformation $\Sigma$ of this group is of the form $T M T^{-1}$ where $T$ is a definite conformal transformation which converts the $\infty^{3}$ circles into our $\Omega$-family and $M$ is any Moebius transformation. This group is generated by conformal symmetries (Schwarzian reflections) with respect to the curves of the $\Omega$ family.

Because of this isomorphism with the Moebius group, we may make the following observations $\left({ }^{1}\right)$.

TheOREM 2. A nonconformal transformation converts at most $2 \infty^{2}$ curves of the given $\Omega$ family into curves of the same family. A conformal transformation, not of the group $G_{6}^{\prime}[\Omega(A, B)]$, converts at most $2 \infty^{1}$ curves of the given $\Omega$ family into curves of the same family.

From this theorem, we may conclude that a point transformation which converts $3 \infty^{2}$ curves of a given $\Omega$ family into curves of the same family must belong to the group $G_{6}^{\prime}[\Omega(A, B)]$. Similarly any conformal transformation which carries $3 \infty^{1}$ curves of a given $\Omega$ family into curves of the same family must belong to the group $G_{6}^{\prime}[\Omega(A, B)]$.

Let $T$ and $R$ be respectively a definite conformal transformation and any other transformation both of which carry the $\infty^{3}$ circles into a given $\Omega(A, B)$ family of curves. Then obviously $R=T M$, where $M$ is a Moebius transformation.

Theorem 1 gives us an analytic characterization of any $\Omega$ family. In the remainder of our work, we shall give geometric characterizations of any such family. For this, we shall suppose that $A$ and $B$ of our family are each not identically zero. Any such family may be obtained by applying to the $\infty^{3}$ circles any conformal transformation written in the form (2) where $\phi$ is not a linear fractional function of $u$ only and $\psi$ is not a linear fractional function of $v$ only.

3. The osculating parabolas of any three-parameter family of curves. Just as a set of values for $\left(u, v, p, p^{\prime}\right)$, that is, a differential element of the second order, is pictured most simply by means of the corresponding circle of curvature, so a differential element of the third order, defined by $\left(u, v, p, p^{\prime}, p^{\prime \prime}\right)$,

(1) Kasner and De Cicco, Characterization of the Moebius group of circular transformations, Proceedings of the National Academy of Sciences, vol. 25 (1939), pp. 209-213. 
may be pictured by the unique osculating parabola. We shall collect here the general formulas to be used in the subsequent discussion $\left({ }^{2}\right)$.

The equation of any parabola in minimal coordinates is

$$
(k u-l v)^{2}+2 k(m+2 l \beta) u+2 l(m+2 k \alpha) v+\left(m^{2}-4 k l \alpha \beta\right)=0,
$$

where $(\alpha, \beta)$ are the minimal coordinates of the focus and $k u+l v+m=0$ is the equation of the directrix. The unique osculating parabola of the differential element of the third order $\left(u, v, p, p^{\prime}, p^{\prime \prime}\right)$ must have the parameters

$$
\begin{aligned}
& k=p p^{\prime \prime}-3 p^{\prime 2}, \quad l=p^{\prime \prime}, \quad m=-3 p p^{\prime}-k u-l v, \\
& \alpha=u+\frac{3 p^{\prime}}{2 p^{\prime \prime}}, \quad \beta=v+\frac{3 p^{2} p^{\prime}}{2\left(p p^{\prime \prime}-3 p^{\prime 2}\right)} .
\end{aligned}
$$

Solving the last two equations for $p^{\prime}$ and $p^{\prime \prime}$, we find

$$
p^{\prime}=\frac{p}{2}\left[\frac{1}{\alpha-u}-\frac{p}{\beta-v}\right], \quad p^{\prime \prime}=\frac{3 p}{4(\alpha-u)}\left[\frac{1}{\alpha-u}-\frac{p}{\beta-v}\right] .
$$

Consider now any triply infinite system of curves, defined by a differential equation of third order

$$
p^{\prime \prime}=f\left(u, v, p, p^{\prime}\right) .
$$

Through a given point in a given direction there pass $\infty^{1}$ curves of the system. Each of these has a definite osculating parabola at the given point. The locus of the foci of these parabolas is termed the focal curve and the envelope of the directrices is called the directorial curve. Thus to each lineal element $E(u, v, p)$ of the plane there corresponds a definite focal curve and a definite directorial curve.

The form of the focal curve depends, of course, upon the form of the differential equation. Since $(u, v, p)$ are fixed, $p^{\prime \prime}$ is a certain function of $p^{\prime}$. Substituting this in (12) and eliminating $p^{\prime}$, we obtain the finite equation of the required locus.

To obtain the finite form of the directorial curve, we proceed as follows. Upon writing the equation of the directrices of the osculating parabolas and differentiating it partially with respect to $p^{\prime}$, we obtain

$$
\begin{aligned}
\left(p f-3 p^{\prime 2}\right)(\alpha-u)+f(\beta-v) & =3 p p^{\prime}, \\
\left(p f_{p^{\prime}}-6 p^{\prime}\right)(\alpha-u)+f_{p^{\prime}}(\beta-v) & =3 p .
\end{aligned}
$$

Solving these for $\alpha$ and $\beta$, we find

(2) Kasner, The trajectories of dynamics, these Transactions, vol. 7 (1906), pp. 401-424. Also see the Colloquium volume by Kasner, Differential-Geometric Aspects of Dynamics, 1913; second edition, 1924. Recent discussion and extensions have been given by Moissiev, Fialkow, and MacColl. 


$$
\begin{gathered}
\alpha=u+\frac{p\left(p^{\prime} f_{p^{\prime}}-f\right)}{p^{\prime}\left(-p^{\prime} f_{p^{\prime}}+2 f\right)}, \quad \beta=v+\frac{p\left(-p p^{\prime} f_{p^{\prime}}+p f+3 p^{\prime 2}\right)}{p^{\prime}\left(-p^{\prime} f_{p^{\prime}}+2 f\right)}, \\
(\beta-v)^{2}-p^{2}(\alpha-u)^{2}=\frac{6 p^{3}\left(-p^{\prime} f_{p^{\prime}}+f+3 p^{\prime 2} / 2 p\right)}{\left(-p^{\prime} f_{p^{\prime}}+2 f\right)^{2}} .
\end{gathered}
$$

The third of these is written for later purposes. The elimination of $p^{\prime}$ from the first two of these equations gives the implicit form of our directorial curve.

4. Characterization by focal curves. We wish to consider the three-parameter families of curves such that the corresponding focal curve of any lineal element $E(u, v, p)$ of the plane is a lemniscate $L$ with $E$ as one of the two orthogonal tangent elements at the node of $L$. Before proceeding with this discussion, it is advisable to give a geometric construction of any lemniscate. Let $C$ and $C^{\prime}$ be any two equal circles which are orthogonal at a point $O$. Draw any line $l$ through $O$ and let $l$ intersect $C$ and $C^{\prime}$ in the two points $Q$ and $Q^{\prime}$ respectively. Let $P$ be any point on $l$ such that the distance $O P$ is the mean proportion between the distances $O Q$ and $O Q^{\prime}$. (We note that the square of $O P$ can have two values, one the negative of the other. But if we are careful to choose the signs of $O P$ and $O Q\left(O Q^{\prime}\right)$ such that the product of $O P$ by $Q P$ $\left(Q^{\prime} P\right)$ is equal to the square of the tangent from $P$ to $C\left(C^{\prime}\right)$, then the square of $O P$ can have only one value.) The set of all such points $P$ forms the lemniscate of Bernoulli.

Let the minimal coordinates of the point $O$ and the centers of $C$ and $C^{\prime}$ be respectively $(u, v),(a, b),[u+i(a-u), v-i(b-v)]$. The equations of $C$ and $C^{\prime}$ are respectively

$$
\begin{aligned}
C: \quad(\alpha-u)(\beta-v) & =(a-u)(\beta-v)+(b-v)(\alpha-u), \\
C^{\prime}: \quad(\alpha-u)(\beta-v) & =i(a-u)(\beta-v)-i(b-v)(\alpha-u),
\end{aligned}
$$

where $(\alpha, \beta)$ are the running minimal coordinates of the points of $C$ or $C^{\prime}$. The equation of our lemniscate $L$ is

$$
(\alpha-u)^{2}(\beta-v)^{2}=i(a-u)^{2}(\beta-v)^{2}-i(b-v)^{2}(\alpha-u)^{2} .
$$

There are $\infty^{4}$ lemniscates in the complex plane. The node of our lemniscate $L$ consists of the two orthogonal tangent elements of $C$ and $C^{\prime}$ at the point $O$. Note that there are four pairs of orthogonal circles which define the same lemniscate $L$.

Upon substituting the values of $\alpha$ and $\beta$ as given by (12) into (17) and noting that

$$
(b-v)^{2} /(a-u)^{2}=p^{2},
$$

we obtain the following proposition.

Theorem 3. Property I. A three-parameter family of curves possesses the 
property that the corresponding focal curve of any lineal element $E$ is a lemniscate $L$ with $E$ as one of the two orthogonal tangent elements at the node of $L$ if and only if its differential equation is of the form

$$
2 p p^{\prime \prime}-3 p^{\prime 2}=\lambda(u, v, p) .
$$

The lemniscates of our family of curves (19) are given by the equation

$$
4 \lambda(\alpha-u)^{2}(\beta-v)^{2}=3 p^{2}\left[(\beta-v)^{2}-p^{2}(\alpha-u)^{2}\right] .
$$

From the above equation, we find that, if $(a, b)$ is the center of any one circle of the four orthogonal pairs of circles which define any one of the lemniscates (20) then

$$
i(a-u)^{2}=3 p^{2} / 4 \lambda, \quad i(b-v)^{2}=3 p^{4} / 4 \lambda .
$$

Let us now consider a three-parameter family of curves with the Property I. Of course any such family is given by a differential equation of the form (19). The focal curve associated to any lineal element $E$ of the plane is a lemniscate. If we keep the point $P$ of $E$ fixed and vary the direction of $E$, the centers of the orthogonal pairs of circles defining these lemniscates will describe a locus. We shall call this locus the central curve of the focal lemniscates associated with the point $P$. By (19) and (21), we obtain the following result.

Theorem 4. Property II. A three-parameter family of curves with the Property I will possess the property that the central curve of the focal lemniscates associated with any point $P$ of the plane is an equilateral hyperbola (eccentricity $e= \pm 2^{1 / 2}$ ) with its center at $P$ if and only if its differential equation is of the form

$$
2 p p^{\prime \prime}-3 p^{2}=2 p^{2}\left(A-B p^{2}\right),
$$

where $A$ and $B$ are arbitrary functions of $u$ and $v$ only.

The equilateral hyperbolas of our family of curves (22) are

$$
i A(a-u)^{2}-i B(b-v)^{2}=\frac{3}{8} .
$$

The four foci of this equilateral hyperbola are

$$
\alpha=u \pm i\left(\frac{3 i}{8 A}\right)^{1 / 2}, \quad \beta=v \pm\left(\frac{3 i}{8 B}\right)^{1 / 2} \text {. }
$$

This immediately yields the following proposition.

TheOREM 5. Property III. A three-parameter family of curves is an $\Omega$ family of curves if it possesses the Properties I, II, and the Property III described as. follows. To any point $P$ of the plane, there is associated by Property II an equilateral hyperbola $H$. The four foci of $H$ are related to the point $P$ by a direct conformal transformation. 
Thus an $\Omega$ family of curves has been completely characterized geometrically by the Properties I, II, and III. In the next section, we shall give an alternate characterization by the means of the focal lemniscates.

5. Alternate characterization by focal lemniscates. Next we shall discuss two Properties, $\mathrm{II}_{1}$ and $\mathrm{III}_{1}$, which are respectively equivalent to Properties II and III. First let us note that a minimal quadrilateral $R$ is any quadrilateral whose sides are minimal lines. The center of $R$ is the intersection of its diagonals. By (19) and (20), we discover the following result.

Theorem 6. Property $\mathrm{II}_{1}$. A three-parameter family of curves with the Property I will possess the property that the $\infty^{1}$ focal lemniscates associated with any point $P$ of the plane all pass through the vertices of a minimal quadrilateral $R$ with center at $P$ if and only if its differential equation is of the form (22).

The four vertices of the minimal quadrilateral of our family of curves (22) are

$$
\alpha=u \pm\left(\frac{3}{8 A}\right)^{1 / 2}, \quad \beta=v \pm\left(\frac{3}{8 B}\right)^{1 / 2}
$$

From this, we find

Theorem 7. Property III $_{1}$. A three-parameter family of curves is an $\Omega$ family of curves if it possesses the Properties $\mathrm{I}_{1} \mathrm{II}_{1}$ and the Property $\mathrm{III}_{1}$ described as follows. To any point $P$ of the plane, there is associated a minimal quadrilateral $R$. The four vertices of $R$ are related to the point $P$ by a direct conformal transformation.

In the above work, we have completely characterized an $\Omega$ family of curves by means of the focal lemniscates. That is, we have given two equivalent sets of geometric characterizations by means of focal lemniscates. In the next section, we shall characterize any such family by means of directorial equilateral hyperbolas.

6. Characterization by directorial curves. In this section, we wish to consider three-parameter families of curves such that the corresponding directorial curve of any lineal element $E(u, v, p)$ of the plane is an equilateral hyperbola $H$ with the point of $E$ as the center of $H$ and the line of $E$ as one of the asymptotes of $H$. The equation of $H$ must be of the form

$$
(\beta-v)^{2}-p^{2}(\alpha-u)^{2}=3 p^{3} / g^{2},
$$

where $g$ is an arbitrary function of $(u, v, p)$ only. Substituting (15) into (26), we find

$$
\frac{-p^{\prime} f_{p^{\prime}}+f+3 p^{2} / 2 p}{\left(-p^{\prime} f_{p^{\prime}}+2 f\right)^{2}}=\frac{1}{2 g^{2}} \text {. }
$$


This is a partial differential equation in the unknown function $f$ whose form is to be determined.

Upon making the transformation

$$
f=\frac{3 p^{\prime 2}}{2 p}+\frac{1}{2}\left(g^{2}-F^{2}\right),
$$

where $F$ is our new unknown function, the differential equation (27) becomes

$$
F^{2}\left[p^{\prime 2} F_{p^{\prime}}^{2}-2 p^{\prime} F F_{p^{\prime}}+F^{2}-g^{2}\right]=0 .
$$

The solution of this yields

$$
F=0, \quad \text { or } \quad F=\mp g+p^{\prime} h,
$$

where $g$ and $h$ are functions of $(u, v, p)$ only. This shows that our differential equation (13) must be either one of the two types

$$
\begin{aligned}
& \mathrm{A}: \quad p^{\prime \prime}=\frac{3 p^{\prime 2}}{2 p}+g^{2} / 2 \\
& \mathrm{~B}: \quad p^{\prime \prime}=\frac{3 p^{\prime 2}}{2 p} \pm g h p^{\prime}-\frac{h^{2}}{2} p^{\prime 2} .
\end{aligned}
$$

The second type $B$ is any three-parameter family of curves whose differential equation is of the form

$$
p^{\prime \prime}=G p^{\prime}+H p^{2}
$$

where $G$ and $H$ are arbitrary functions of $(u, v, p)$ only. Any such family may be characterized by the property that the focal curve at any element $E$ is a circle passing through the point of $E$. This type has been considered extensively by Kasner in connection with his geometry of dynamical trajectories (first of Kasner's five dynamical properties( $\left.{ }^{2}\right)$ ).

Upon substituting the second of equations (31) into (14) and (15), we discover that the directrices of the $\infty^{1}$ osculating parabolas form a pencil of straight lines with the vertex

$$
\alpha=u \pm \frac{3-h^{2} p}{2 g h}, \quad \beta=v \pm \frac{\left(3+h^{2} p\right) p}{2 g h} .
$$

Thus for this second type B our equilateral hyperbola is degenerate. As a matter of fact, it may be proved $\left({ }^{2}\right)$ that any three-parameter family of curves for which the $\infty^{1}$ directrices of the osculating parabolas at any element of the plane form a pencil of straight lines is given by a differential equation of the form (32).

The first type (A) of (31) is any three-parameter family of curves whose differential equation is of the form (19). Any such family is characterized by 
the Property I. Upon substituting the first of equations (31) into (14) and (15), we find that our directorial curve is a nondegenerate equilateral hyperbola as given by (26). By comparing the second of equations (31) with (19), we find $\lambda=p g^{2}$. Our equilateral hyperbola (26) then becomes

$$
(\beta-v)^{2}-p^{2}(\alpha-u)^{2}=3 p^{4} / \lambda .
$$

TheOREM 8. Property I'. A three-parameter family of curves for which the directorial curve at any element $E$ is a nondegenerate equilateral hyperbola $H$ with the point of $E$ as the center of $H$ and the line of $E$ as one of the asymptotes of $H$ is given by a differential equation of the form (19). Thus Property $\mathrm{I}^{\prime}$ is equivalent to Property I.

From (34), we find that if $(\alpha, \beta)$ denotes any focus of our equilateral hyperbola $H$, then

$$
\begin{gathered}
(\alpha-u)^{2}=-3 p^{2} / \lambda, \quad(\beta-v)^{2}=3 p^{4} / \lambda, \\
A(\alpha-u)^{2}+B(\beta-v)^{2}=-3 p^{2}\left(A-B p^{2}\right) / \lambda,
\end{gathered}
$$

where $A$ and $B$ depend only on $(u, v)$. From the above equations, we deduce the following result.

Theorem 9. Property II'. A three-parameter family of curves with the Property $I^{\prime}$ will possess the property that the curve of foci of the directorial equilateral hyperbolas associated with any point $P$ of the plane is an equilateral hyperbola $H^{\prime}$ with center at $P$ if and only if its differential equation is of the form (22). Thus Property II' $^{\prime}$ is equivalent to Property II.

The equilateral hyperbolas of Theorem 9 are

$$
A(\alpha-u)^{2}+B(\beta-v)=-\frac{3}{2} .
$$

Next if $(\alpha, \beta)$ denotes the center of any one of the four pairs of orthogonal circles which define any one of the above lemniscates, then

$$
\alpha=u \pm i\left(\frac{3}{2 A}\right)^{1 / 2}, \quad \beta=v \pm i\left(\frac{3}{2 B}\right)^{1 / 2} \text {. }
$$

THEOREM 10. Property III'. A three-parameter family of curves is an $\Omega$ family of curves if it possesses the Properties $\mathrm{I}^{\prime}, \mathrm{II}^{\prime}$, and the Property III' described as follows. To any point $P$ of the plane, there is associated by Property II' an equilateral hyperbola $H^{\prime}$. The four foci of $H^{\prime}$ are related to the point $P$ by $a d i$ rect conformal transformation. Thus Property III' is equivalent to Property III.

In this section, we have completely characterized an $\Omega$ family of curves with a third set of geometric properties. This set depends essentially on the directorial equilateral hyperbolas. In the next section, we shall give some additional geometric properties of such a family. 
7. Additional geometric properties of an $\Omega$ family. Upon replacing $\lambda$ by the value $2 p^{2}\left(A-B p^{2}\right)$ in (34), we find that the envelope of the $\infty^{1}$ equilateral hyperbolas (34) is

$$
\left[A(\alpha-u)^{2}+B(\beta-v)^{2}+\frac{3}{2}\right]^{2}=4 A B(\alpha-u)^{2}(\beta-v)^{2} .
$$

From this, we derive the following result.

THEOREM 11. An $\Omega$ family of curves possesses the following additional property. The envelope of the $\infty^{1}$ directorial equilateral hyperbolas of the lineal elements through a fixed point $P$ consists of the four sides of a rectangle $R$ whose diagonals are the minimal lines through $P$. Moreover, the vertices of this rectangle $R$ are connected to $P$ by a direct conformal transformation.

Next we note that the two sets of geometric characterizations (I, II, III) and $\left(\mathrm{I}^{\prime}, \mathrm{II}^{\prime}, \mathrm{III}^{\prime}\right)$ give rise to a reciprocity relation. Let us consider as reciprocal elements: the focus and directrix of our osculating parabola; a lemniscate $L$ and an equilateral hyperbola $H$ with the node of $L$ and the center of $H$ coincident; the tangent lines of the node of $L$ and the asymptotes of $H$. Of course, the centers of the orthogonal pairs of circles defining $L$ and the foci of $H$ both describe equilateral hyperbolas, which are equivalent by a similitude. Then we observe that the two sets of geometric characterizations (I, II, III) and $\left(\mathrm{I}^{\prime}, \mathrm{II}^{\prime}, \mathrm{III}^{\prime}\right)$ are reciprocal. This reciprocity is brought to light more by the following two results.

Theorem 12. The focal lemniscate $L$ (20) of Property I and the directorial equilateral hyperbola $H$ (34) of Property $\mathrm{I}^{\prime}$ are equivalent under the Moebius inversion with respect to the circle with center at the point $P$ and with radius $R^{2}=3 p^{3} / 2 \lambda$.

Theorem 13. The equilateral hyperbolas $H$ (23) of Property II and $H^{\prime}$ (36) of Property $\mathrm{II}^{\prime}$ are equivalent under the similitude

$$
\alpha^{\prime}-u= \pm 2 i^{3 / 2}(\alpha-u), \quad \beta^{\prime}-v= \pm 2 i^{1 / 2}(\beta-v) .
$$

This similitude can be factored in to the product of the rotation about the point $P$ through the angle $\pi / 4+k \pi / 2$ by the magnification through $P$ of ratio \pm 2 or $\pm 2 i$ according as $k$ is odd or even.

In the next section, we shall give a fourth and final geometric characterization of an $\Omega$ family of curves by means of the rate of variation of curvature with respect to the arc length.

8. Characterization by the rate of variation of curvature. The curvature $\kappa$ and the rate $d \kappa / d s$ of variation of the curvature with respect to the arc length $s$ are given in minimal coordinates by

$$
2 i_{\kappa}=\frac{p^{\prime}}{p^{3 / 2}}, \quad 2 i \frac{d \kappa}{d s}=\frac{2 p p^{\prime \prime}-3 p^{\prime 2}}{2 p^{3}} .
$$


From these, we derive the following.

THEOREM 14. Property $\mathrm{I}^{\prime \prime}$. A three-parameter family of curves for which the rate $d \kappa / d s=\kappa^{\prime}$ of variation of the curvature per unit length of arc is the same for the $\infty^{1}$ curves of the family at any lineal element $E$ must have its differential equation of the form (19). Thus the Properties I, I', I" are all equivalent.

Let a three-parameter family of curves possess the Property $\mathrm{I}^{\prime \prime}$. Then to each lineal element of the plane, there is associated a definite rate of variation of curvature. That is, $\kappa^{\prime}=\kappa^{\prime}(E)$.

TheORem 15. Property II". Consider any three-parameter family of curves with the Property $\mathrm{I}^{\prime \prime}$. Let there exist an orthogonal net of curves $N$ with the following property. At any point $P$ of the plane, construct the two orthogonal lineal elements $E_{j}(j=1,2)$ which belong to $N$. The rate $\kappa^{\prime}(E)$ of variation of curvature of any element $E$ through $P$ is proportional to the cosine of twice the angle between $E$ and $E_{j}$. Any three-parameter family with this additional property must be given by a differential equation of the form (22).

The Property II" of the preceding theorem is an analogue of the Meusnier theorem for curves on a surface in three-space. This property may be written in the form

$$
\kappa^{\prime}=\kappa_{i}^{\prime} \cos 2 \theta,
$$

where $\theta$ is the angle between the two lineal elements $E$ and $E_{j}$ at the point $P$ and $\kappa^{\prime}$ and $\kappa_{j}^{\prime}$ are the rates of variation of curvature at $E$ and $E_{j}$ respectively.

The differential equation of the net $N$ and the value of $\kappa_{j}^{\prime}$ are respectively

$$
p_{j}= \pm i\left(\frac{A}{B}\right)^{1 / 2}, \quad \kappa_{j}^{\prime}=\mp(A B)^{1 / 2} .
$$

By this, we may state simply

Theorem 16. Property.III". A three-parameter family of curves is an $\Omega$

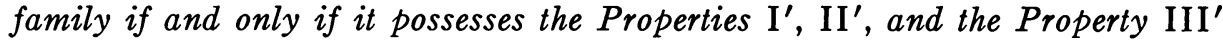
described as follows. The function $\kappa_{j}^{\prime} p_{j}$ depends on $u$ only and the function $\kappa_{j}^{\prime} / p_{j}$ depends on $v$ only.

This completes our list of geometric characterizations of an $\Omega$ family. We have completely listed four such sets of characterizations, namely: Properties (I, II, III); (I, $\left.\mathrm{II}_{1}, \mathrm{III}_{1}\right)$; $\left(\mathrm{I}^{\prime}, \mathrm{II}^{\prime}, \mathrm{III}^{\prime}\right)$; and $\left(\mathrm{I}^{\prime \prime}, \mathrm{II}^{\prime \prime}, \mathrm{III}^{\prime \prime}\right)$.

9. The hyperosculated isothermal net of an $\Omega$ family. From Theorem 16, we deduce that the orthogonal net $N$ of Theorem 15 is an isothermal net. But this property is not sufficient to characterize an $\Omega$ family of curves. Next we make the following observation. 
THEOREM 17. All the curves of an $\Omega$ family which are hyperosculated by their osculating circles form an isothermal net. This net cuts the isothermal net $N$ of Theorems 15 and 16 at an angle of $\pi / 4$ radians $\left(45^{\circ}\right)$.

This last theorem is of significant interest for the following reasons. Let us define a conformal transformation, not of the Moebius group, which preserves the maximum number of circles to be a conformal near-Moebius transformation $\left({ }^{3}\right)$. Under any such transformation $\Sigma$, it results that the only possible circles that are preserved must form the hyperosculated isothermal net of the $\Omega$ family into which the $\infty^{3}$ circles are converted by $\Sigma$. It is known that the only isothermal circular nets are two orthogonal pencils of circles. The conformal near-Moebius transformations are deducible from this result. These are of the form $\Sigma=M_{2} T M_{1}$ where $M_{1}$ and $M_{2}$ are Moebius transformations and $T$ is one of the three transformations

$$
U=u^{n}, \quad V=v^{n} ; \quad U=e^{u}, \quad V=e^{v} ; \quad U=\log u, \quad V=\log v .
$$

As a corollary of this work, we may state

THEOREM 18. The only $\Omega$ family of curves (not circles) of the $(u, v)$ plane which contain circles as the hyperosculated isothermal net are

$$
\begin{aligned}
& a U^{n} V^{n}+b U^{n}+c V^{n}+d=0, \\
& a e^{U+V}+b e^{U}+c e^{V}+d=0, \\
& a \log U \log V+b \log U+c \log V+d=0,
\end{aligned}
$$

where $(U, V)$ are Moebius functions of $(u, v)$.

In the present paper, we have completely characterized the $\Omega$ families of curves. One analytic and four geometric characterizations, each consisting of three independent properties, have been given. In later work, we shall study certain subfamilies $\left({ }^{4}\right)$ of any $\Omega$ family and then develop the geometry of these families with respect to Schwarzian reflection (conformal symmetry) $\left(^{5}\right)$. We state in conclusion

TheOREM 19. Every horn angle contained in an $\Omega$ family is conformally equivalent to a circular horn angle, and therefore its measure is $M=\infty$.

This property belongs to a much larger category of families. The conformal measure of a horn angle is defined as

(3) Kasner and De Cicco, The conformal near-Moebius transformations, Bulletin of the American Mathematical Society, vol. 46 (1940), pp. 784-793.

(4) This is related to the theory of natural families. See papers by Kasner (1909), Lipke (1912), Douglas (1924), Fialkow (1939), and Struik's recent treatise on differential geometry (1938) where other references can be found.

(5) Kasner, Annals of Mathematics, (2), vol. 38 (1937), pp. 873-877. 


$$
M_{12}=\left(\gamma_{2}-\gamma_{1}\right) /\left(\frac{d \tau_{2}}{d s}-\frac{d \gamma_{1}}{d s}\right)
$$

where $\gamma$ denotes curvature. If we demand that this shall be constant, a very extensive type of triple family is obtained.

Columbia University, NEW YORK, N. Y.

ILLINOIS INSTITUTE OF TEChNOLOGY, Chicago, Ill. 